\title{
CD95L pro-drug: a novel Swiss Army Knife in cancer therapy?
}

\author{
D Kassahn ${ }^{1}$, U Nachbur ${ }^{1}$ and T Brunner ${ }^{\star, 1}$
}

In the search for the 'magic bullet' to fight cancer, scientists have developed a plethora of more or less useful drugs and treatments. Nonetheless, the current success rate of various antitumor chemotherapeutics and biologics for the noninvasive treatment is still quite disappointing. A major problem of most noninvasive treatments of tumors is the fact that chemotherapeutics and biologics are not very tumor-specific, but cause a lot of damage to normal untransformed cells and thereby severe side effects. This is particularly true for death ligand (TNF $\alpha$, CD95L and TRAIL)-based biologics, which besides inducing apoptosis in tumor cells have a broad spectrum of effects on normal cells. ${ }^{1}$ An ideal anticancer drug would thus be selective and specific for tumor cells, while not causing any damage to untransformed cells.

In this issue of CDD, Watermann and colleagues describe a novel and promising approach as to how death ligands can be kept on a tight leash and be released on command. This novel recombinant CD95L-based pro-drug appears to fulfill many principal criteria of a tumor-specific therapy: systemic safety, tumor-specific targeting and activation of the pro-drug and efficacy.

The basis of the safety of this pro-drug is the neutralization and inactivation of recombinant CD95L by its cognate receptor CD95. Receptor and ligand are integrated within the same fusion protein and separated by a proteasesensitive site. CD95L remains inactive until released from its leash (the CD95 receptor) by tumor-expressed proteases. Normal tissue cells are not 'seen' by the CD95L pro-drug, and therefore it can be administered systemically. To link CD95L with its receptor, the authors have chosen an MMP2-sensitive sequence, as this matrix metalloprotease is frequently overexpressed on a number of tumor cells. ${ }^{2}$ Intriguingly, however, cell surface expression of MMP2 alone is insufficient to activate the CD95L pro-drug, and closer proximity appears to be required. This is achieved by the last tool of this multifunctional construct, a tumor antigen-specific singlechain antibody. The sequence of pro-drug targeting and activation is illustrated in Figure 1.

In this study, the authors show convincingly that the CD95L pro-drug is devoid of any nonspecific toxicity and is only activated on tumor cells expressing (i) the tumor antigen and (ii) the proper protease to activate the pro-drug. Upon binding of the single-chain antibody to the tumor antigen, the tumor protease cleaves the protease-sensitive spacer and releases
CD95L from its receptor. Interestingly, the recombinant soluble receptor seems to be incapable of competing with the more abundant cell surface receptors on tumor cells, and apoptosis is efficiently induced. Most likely the clustering and aggregation of recombinant CD95L on the target cell by the single-chain antibody further potentiates its apoptosisinducing activity, as both clustering of CD95 and CD95L, for example, by membrane rafts or antibodies, enhances their apoptosis-inducing activities. ${ }^{3}$ Critically, despite the potent death-inducing activity of this drug in tumor cells in vitro and in vivo, no side effects on tissue cells have been observed when applied systemically. This is a major improvement over previous CD95L-based antitumor drugs. Even low doses of conventional recombinant $\mathrm{CD} 95 \mathrm{~L}^{4}$ or agonistic anti-CD95 antibodies ${ }^{5}$ cause massive liver destruction and even death, excluding their systemic administration. The novelty of the current approach is therefore not only that it specifically targets CD95L to the tumor cell via the single-chain antibody, but in particular that it allows activation of the CD95L only on the tumor cell by keeping it in check until the tumor protease removes the neutralizing CD95 receptor. In particular, this safety issue has not been fully achieved by previous attempts to deliver bi-specific anti-CD95/anti-tumor antibodies ${ }^{6}$ or adenovirus-encoded CD95 $\mathrm{L}^{7}$ to tumor cells. Most astonishing is the fact that Watermann and colleagues managed to pack all of these different domains and functions into one fusion protein, very much like a Swiss Army Knife. In summary, with this tumor cell-activated CD95L the authors have developed a novel and multifunctional drug with yet unforeseen possibilities in cancer therapy.

Although this novel death ligand-based drug with its selfcontrolled approach appears promising, it may also have its limits. In order to work properly, different parameters have to be fulfilled. Tumor cells must express the tumor antigen, the protease and the death receptor at sufficiently high levels to allow targeting, activation and apoptosis induction. Most important, the tumor cell must be CD95L-sensitive. This is likely a critical parameter as tumor cells have acquired a plethora of mechanisms to avoid apoptosis induction, including the upregulation of antiapoptotic molecules and the downregulation of death receptors. ${ }^{8} \mathrm{~A}$ tumor cell that can activate the pro-drug owing to the expression of the tumor antigen and the appropriate protease, but does not die, may accumulate active CD95L on the cell surface. This in turn 

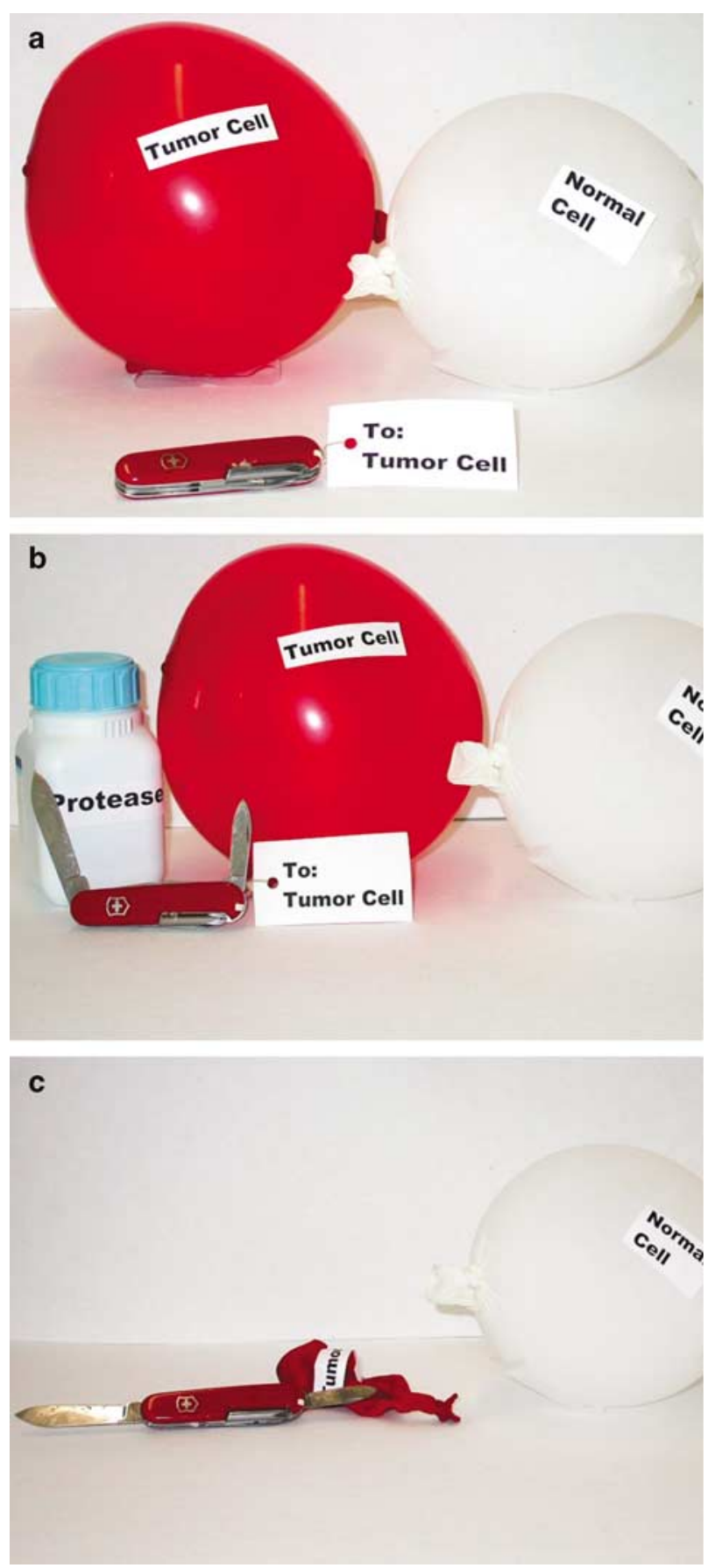

Figure 1 Targeted activation of the CD95L pro-drug. (a) The inactive CD95L prodrug is selectively targeted to the tumor cell by its tumor antigen-specific singlechain antibody. The pro-drug fails to bind to nontumor cells. (b) Upon binding, tumor-specific proteases activate the CD95L pro-drug. (c) Active CD95L binds to cell surface CD95 and induces apoptosis in the target cell. Nontumor cells remain intact could be used by the tumor cell to induce apoptosis in tumorinfiltrating cytotoxic $T$ cells and thus escape from the antitumor immune response. ${ }^{9} \mathrm{~A}$ combination therapy may be a good approach to avoid such problems. Chemotherapeutics are known to sensitize tumor cells to death ligand-induced apoptosis by downregulation of antiapoptotic molecules and upregulation of functional death receptors, for example, CD95 and TRAIL-R2. ${ }^{10}$ Last but not least, the pro-drug in its current form is likely to induce an immune response and the generation of neutralizing antibodies, which will limit the prodrug's activity upon repeated treatments. Thus, a CD95Lbased pro-drug may have to be further refined before (pre-) clinical trials in patients.

While it is likely still a long way to the perfect tumor therapy, this novel Swiss Army Knife (which in this case is of German origin) introduces a novel and most promising concept of targeted cancer treatment with reduced side effects.

1. Locksley RM et al. Cell 2001; 104: 487-501.

2. Overall CM, Dean RA. Cancer Metastasis Rev 2006; 25: 69-75.

3. Nachbur U et al. Blood 2006; 107: 2790-2796.

4. Loo G et al. Cytokine 2003; 22: 62-70.

5. Ogasawara J et al. Nature 1993; 364: 806-809.

6. Jung $\mathrm{G}$ et al. Cancer Res 2001; 61: 1846-1848.

7. Aoki $\mathrm{K}$ et al. $\mathrm{Mol}$ Ther 2000; 1: 555-565.

8. Hopkins-Donaldson S et al. Cell Death Differ 2003; 10: 356-364.

9. Cefai D et al. Cell Death Differ 2001; 8: 687-695.

10. el-Deiry WS. Semin Cancer Biol 1998; 8: 345-357. 